\title{
Genetic Evaluation of Tomato (Solanum lycopersicum L.) Germplasm for Yield and Quality Traits
}

\author{
Dharminder Kumar ${ }^{1}$, Achal Kashyap ${ }^{2}$, Balbir Singh Dogra ${ }^{2}$, Kumud Jaryal ${ }^{2}$, Atul \\ Gupta $^{1}$, Sandeep Kumar ${ }^{4}$, Aanchal Chauhan ${ }^{3}$, Kumari Shiwani ${ }^{3}$ and Rajesh Kaler ${ }^{1}$ \\ ${ }^{1}$ RHR \& TS Jachh, The Nurpur, District Kangra (H.P) 176201, India \\ ${ }^{2}$ YSP UHF, College of Horticulture and Forestry, Neri, Hamirpur, 177001, India \\ ${ }^{3}$ College of Horticulture and Forestry, Thunag, Mandi, 175048, India \\ ${ }^{4}$ ICAR-IARI, RS-Katrain, Kullu Valley, 175129, India \\ *Corresponding author
}

\section{A B S T R A C T}

\section{Keywords}

Genetic advance,

Genotypes,

Heritability,

Variability,

Tomato,

Yield

Article Info

Accepted:

04 October 2020

Available Online:

10 November 2020
Genetic evaluation of twenty five diverse genotypes tomato was conducted for yield and quality traits at the experimental farm of RHR\&TS, Jachh, Kangra, Dr YS Parmar University of Horticulture and Forestry, Nauni, Solan, Himachal Pradesh during the year 2018. These genotypes were comprised of eleven varieties, ten exotic lines and three local collections along with one standard check Solan Lalima. Experiment was conducted in Randomized Complete Block Design with three replications to ascertain extent of variability, heritability, genetic advance and gain for yield and other horticultural traits. Analysis of variance revealed significant differences among all the genotypes for all the characters under study. The estimates of PCV and GCV were high for marketable fruit yield per plant, number of locules per fruit, average fruit weight, harvest duration, shelf life and number of fruits per cluster. High heritability estimates were observed for all the traits among all genotypes while high estimates of genetic gain were observed for marketable fruit yield per plant, average fruit weight, harvest duration, shelf life, number of fruits per cluster, number of locules per fruit, number of fruits per plant, fruit breadth, pericarp thickness, number of fruit cluster per plant, fruit length and plant height. On the basis of overall performance, EC-802557, LC-2 and Arka Alok were found superior for marketable fruit yield and other important horticultural traits. They could be the promising parents for utilization in further breeding programmes.

\section{Introduction}

Tomato is a very popular warm season vegetable crop grown worldwide because it has wider adaptation along with high yield potential and is suitable for fresh use as well as in processed form. It ranks second only after potato (Bose et al., 2002). It is designated as "Protective food" because of its nutritive value and presence of several 
antioxidants viz. carotenoids, particularly lycopene, ascorbic acid, vitamin E and phenol compounds, particularly flavonoids. Lycopene is reported to possess dietetic properties as it reduces the risk of heart attacks and various types of cancers (Clinton, 2005). In India, it is cultivated over an area of nearly 814 thousand ha with annual production of 20,515 thousand MT [Anonymous, 2018-19 (a)]. Madhya Pradesh, Andhra Pradesh, Karnataka, Odisha are the major producing states of tomato in India. It has the share of nearly $8.3 \%$ of total vegetable area and $10.3 \%$ of total vegetable production in India. Though, tomato is one of the major vegetables exported from India, per capita consumption in India is abysmally low. In the year 2014-15, an estimated 0.22 MT of tomato worth 44, 461.34 Lakh were exported from India.

Himachal Pradesh is a major off-season tomato growing state covering an area of 9.93 thousand hectare with an annual production of 4.13 lakh metric tonnes and productivity of 41.7 metric tonnes per hectares. Considering the importance of this crop, there is an urgent need for development of new high yielding varieties/hybrids of tomato with good quality attributes. A complete knowledge pertaining to the amount of genetic variability existing for different traits is considered a prerequisite to start any crop improvement programme. The phenotypic expression of a particular trait is mainly influenced by the genetic makeup of the plant and the environment, in which it is growing. Further, the genetic variance of any quantitative trait is comprised of additive and non-additive variance. Therefore, it become pertinent that observed phenotypic variability is sub-divided into its heritable and nonheritable components with the help of suitable parameters viz. coefficients of variation, heritability and genetic advance. Further, genetic advance can be used to predict the efficiency of selection. Therefore, the present investigation was undertaken with the objective to estimate the extent of genetic variability for yield and quality traits in different genotypes of tomato

\section{Materials and Methods}

The experimental trial was carried out during 2018 at Regional Horticultural Research and Training Station, Dr YS Parmar University of Horticulture and Forestry, Jachh (Nurpur), District-Kangra, Himachal Pradesh. This site is situated on Pathankot-Mandi National Highway (NH-22) at an altitude of $428 \mathrm{~m}$ above mean sea level, lying between $32^{0} 16$ 54.02" N latitude and 75 51 '4.38" E longitude under sub-mountain and low hill sub- tropical agro-climatic zone of Himachal Pradesh, India. The mean annual rainfall received by this area was $1500 \mathrm{~mm}$. The soil structure of the experimental farm was sandy loam to clay loam with the $\mathrm{pH}$ ranging from 6.8-7.0. The complete lists of genotypes along with their source of collection have been presented in Table 1.

The seed sowing of all the genotypes were carried out during March, 2018 in raised nursery bed. Recommended cultural practices were followed for raising the healthy nursery. The experiment was laid out in the Randomized Complete Block Design (RCBD) with three replications during April, 2018. The experiment was laid out in layout are given below Randomized Complete Block Design (RCBD) with three replications. After raising healthy nursery it was transplanted in the plots of $1.8 \mathrm{~m} \mathrm{x} 1.8 \mathrm{~m}$ at a spacing of $60 \mathrm{~cm} \mathrm{x} 45$ $\mathrm{cm}$. Observations were recorded for various growth and fruit yield characteristics like days to first fruit harvesting (number), plant height $(\mathrm{cm})$, fruit length $(\mathrm{cm})$, fruit breadth $(\mathrm{cm})$, average fruit weight $(\mathrm{g})$, number of fruit clusters per plant, number of fruits per cluster, number of fruits per plant, number of locules per fruit, harvest duration (days), marketable fruit yield per plant $(\mathrm{kg}) \&$ per 
hectare (q) and fruit quality characteristics like shelf life (days), pericarp thickness (mm), total soluble solids ( ${ }^{\mathrm{B}} \mathrm{Brix}$ ), For each observed character, the statistical analysis was performed by using MS-Excel, OPSTAT and SPAR 1.0 packages. Analysis of variance (ANOVA) was done by the method recommended by Gomez and Gomez (1983) for Randomized Complete Block Design.

All the characters which showed significant differences among genotypes were further subjected to analysis for the following parameters like coefficients of variability (phenotypic and genotypic), heritability, genetic advance and genetic gain. Variability at genotypic (GCV) and phenotypic (PCV) levels were estimated as per the method suggested by Burton and De- Vane (1953).

Heritability in broad sense was estimated as per the formulae suggested by Allard (1960). The expected genetic advance (GA) resulting from selection of five percent superior individuals was calculated as per Allard (1960). Genetic gain expressed as per cent ratio of genetic advance and population mean was calculated by the method given by Johnson et al., (1955). The genetic advance as per cent over mean was calculated as mentioned by Johnson et al., 1955.

\section{Results and Discussion}

\section{Variability studies}

\section{Mean performance of genotypes}

A highly significant difference was observed among the genotypes for all the traits studied which indicated the existence of enough variability in the germplasm under study. The mean performance of twenty-five genotypes along with parameters of variability for different horticultural traits are described and discussed below:

\section{Days to first fruit harvesting (number)}

Its value ranged from (62.00 - 81.33 days $)$ with a grand mean of 69.69 days (Table 2). Thirteen genotypes including check were earlier in days to first fruit harvesting than the total population mean. Minimum number of days to first fruit harvesting was recorded in LC-2 (62.00 days) and maximum number of days to first fruit harvesting was recorded in Pusa Rohini (81.33 days). Considerable variability regarding this trait was found in accordance with the findings of Rai et al., (2016) and Patel et al., (2013).

\section{Plant height (cm)}

Wide variation observed among all the genotypes for plant height. The overall mean value for the trait was $71.57 \mathrm{~cm}$ (Table 2). It varied from 55.73 to $98.87 \mathrm{~cm}$. Ten genotypes including check had more plant height than the population mean $(71.57 \mathrm{~cm})$. Maximum plant height was recorded in Pusa Ruby (98.87 $\mathrm{cm})$ whereas minimum plant height was recorded in Arka Alok $(55.73 \mathrm{~cm})$. Considerable variation for this trait was also observed by Meena et al., (2018) and Singh et al., (2017a).

\section{Fruit length (cm)}

A significant variation was observed among all the genotypes under study for fruit length. It ranged from 3.53 to $5.89 \mathrm{~cm}$ (Table 2). Sixteen genotypes including standard check (Solan Lalima) produced longer fruits than population mean $(4.97 \mathrm{~cm})$. EC- 802562 (5.89 $\mathrm{cm})$ reported the maximum fruit length and was found statistically at par with 5 genotypes viz. EC-802563 (5.87 cm), EC-802561 (5.63 $\mathrm{cm})$, EC-802557 (5.57 cm), Punjab Chhuhara $(5.61 \mathrm{~cm})$ and Arka Vikas $(5.63 \mathrm{~cm})$ whereas, minimum fruit length was observed in EC$802555(3.53 \mathrm{~cm})$ and was found statistically at par with Pant T-3 $(3.79 \mathrm{~cm})$ and Pusa Ruby 
$(3.58 \mathrm{~cm})$. Among all the genotypes under study, seven genotypes had larger fruit length than standard check Solan Lalima $(5.43 \mathrm{~cm})$. Meena et al., (2018) and Prajapati et al., 2015 reported a wide variations for fruit length.

\section{Fruit breadth $(\mathrm{cm})$}

Fruit breadth ranged from 2.64 to $5.96 \mathrm{~cm}$ (Table 2) and varied significantly among all the genotypes under study as shown in Table 1. Sixteen genotypes including standard check had higher fruit breadth than population mean $(4.99 \mathrm{~cm})$. Highest breadth was observed in Arka Alok $(5.96 \mathrm{~cm})$ and was statistically at par with 3 genotypes viz. LC-2 $(5.75 \mathrm{~cm})$, EC$802557(5.70 \mathrm{~cm})$ and EC-802562 $(5.65 \mathrm{~cm})$. Minimum fruit breadth was recorded in LC-3 $(2.64 \mathrm{~cm})$. Twelve genotypes under study had greater fruit breadth than standard check Solan Lalima $(5.23 \mathrm{~cm})$. Similar findings were also reported by Meena et al., (2018), Prajapati et al., 2015.

\section{Average fruit weight (g)}

Average fruit weight ranged from 25.37 to $76.65 \mathrm{~g}$ (Table 2) and varied significantly among all the genotypes under study. Highest average fruit weight was observed in EC802562 (76.65 g) and was statistically at par with Arka Alok (75.62 g) and EC-802563 $(74.91 \mathrm{~g})$. Minimum average fruit weight was recorded in LC-3 (25.37 g) and was statistically at par with EC-802555 (30.08 g).

Seven genotypes viz., EC- 802563 (74.91 g), EC-802562 (76.65 g), EC-802561 (70.44 g), EC-802557 (70.17 g), Arka Vikas (68.85g), Arka Alok (75.62 g) and LC-2 967.35 g) gave higher fruit weight than the standard check cultivar Solan Lalima (65.32 g). It is one of the major yields contributing character. Wide genetic variation with respect to this character was also reported by Meena et al., (2018), Prajapati et al., 2015 and Patel et al., (2013).

\section{Number of fruit clusters per plant}

The number of fruit cluster per plant varied from 3.60 to 7.20 (Table 3) and varied significantly among all the genotypes under study (Table 2). Eleven genotypes had more number of fruits per cluster than population mean. EC-802563 (7.20) reported the maximum number of fruits per cluster and was found statistically at par with EC-802562 (6.87) while Pusa Uphar (3.60) reported the minimum number of fruit cluster per plant. Twenty-one genotypes produced more number of fruit cluster per plant than the standard check cultivar Solan Lalima (5.10). Results of the present study were in line with the findings of Meena et al., (2018) and Reddy et al., (2013a).

\section{Number of fruits per cluster}

The number of fruits per cluster varied from 2.31 to 5.65 with the overall mean 3.76 (Table 2) and varied significantly among all the genotypes under study. Twelve genotypes including standard check had more number of fruits per cluster than population mean. Maximum number of fruits per cluster was observed in Pusa Uphar (5.65) while EC802563 (2.31) reported the minimum number of fruits per cluster and was found statistically at par with EC-802559 (2.58). Results of the present study were in line with the findings of Meena et al., (2018), Rai et al., (2016), Prajapati et al., (2015) and Shankar et al., (2013).

\section{Number of fruits per plant}

The number of fruits per plant ranged from 15.53 to 30.60 and varied significantly among all the genotypes under study (Table 2). Including standard check twelve genotypes produced more number of fruits per plant than the population means (20.93). Maximum number of fruits per plant was observed in 
genotype EC-802555 (30.60) while LC- 1 (15.53) recorded in minimum number of fruits per plant and was statistically at par with LC3 (17.07), EC-802558 (17.57), EC-802559 (17.02), EC-802561 (15.84) and EC- 802563 (16.62). Eleven genotypes among study produced more number of fruits per plant than the standard check (Solan Lalima, 22.58).

Results of the present study were in line with the findings of Meena et al., (2018), Singh et al., (2017a), Rai et al., (2016), Patel et al., (2013) and Reddy et al., (2013a).

\section{Number of locules per fruit}

Number of locules ranged from 2.33 to 5.67 (Table 3) and varied significantly among all the genotypes under study. EC-802555 (2.33) recorded the minimum number of locules per fruit whereas maximum number of locules per fruit was observed in Arka Abha (5.67).

Eighteen genotypes had reduced number of locules per fruit than the check cultivar Solan Lalima (4.33). Similar results have also been obtained by Meena et al., (2018) and Singh et al., (2017a).

\section{Harvest duration (days)}

Harvest duration ranged from 21.33 to 46.67 days with the overall mean of 31.08 days (Table 3) and varied significantly among all the genotypes under study. Eleven genotypes including standard check had the longer harvest duration than population mean among all the genotypes under study.

Genotype EC-802555 (46.67 days) recorded the maximum harvest duration whereas, EC802563 (21.25 days) reported the minimum harvest duration which was found statistically at par with EC-802561 (23.00 days). Similar results were also reported by Rai et al., (2016) for this attribute.

\section{Shelf life (days)}

Statistical analysis revealed that genotypes showed significant variations for shelf life. It ranged from 14.00 to 31.33 days (Table 3). Overall mean for the character was 22.13 days. Ten genotypes were found to have longer shelf life than population mean.

Maximum shelf life was recorded in genotype EC-802562 (31.33 days) which was found statistically at equivalence with EC-802557 (30.33 days), whereas, minimum shelf life was observed in LC-3 (14.00 days) which was found statistically at par with Pant T-3 (16.00 days). Results of the present study were in accordance with the findings of Reddy et al., (2013a).

\section{Pericarp thickness (mm)}

Pericarp thickness ranged from 4.24 to 7.85 $\mathrm{mm}$ with the mean value $6.46 \mathrm{~mm}$ and varied significantly among all the genotypes under study. Fourteen genotypes including standard check had higher pericarp thickness than population mean. Maximum pericarp thickness was recorded in EC-802562 (31.33 days) which was statistically at par with EC$802557(7.78 \mathrm{~mm})$ and EC-802554 (7.37 $\mathrm{mm})$. Minimum pericarp thickness was observed in LC-3 (4.24 mm). Results of the present study were in line with the findings of Singh et al., (2017a), Rai et al., (2016), Prajapati et al., (2015) and Patel et al., (2013).

\section{Total soluble solids ( ${ }^{\circ}$ Brix)}

Total soluble solids ranged from 3.65- $5.10{ }^{\circ} \mathrm{B}$ and varied significantly among all the genotypes under study. LC-2 $\left(5.10{ }^{\circ} \mathrm{B}\right)$ reported with maximum value for total soluble solids which was statistically at par with Pusa Rohini $\left(4.83{ }^{\circ} \mathrm{B}\right)$, Arka Abha $\left(5.00{ }^{\circ} \mathrm{B}\right)$ and minimum value was recorded in Pusa Ruby $\left(3.65^{\circ} \mathrm{B}\right)$. 
Table.1 List of different genotypes of tomato and their source of collection

\begin{tabular}{|c|c|c|}
\hline Sr. No. & Genotype & Source \\
\hline 1 & EC-802554 & AVRDC, Taiwan \\
\hline 2 & EC-802555 & AVRDC, Taiwan \\
\hline 3 & EC-802556 & AVRDC, Taiwan \\
\hline 4 & EC-802557 & AVRDC, Taiwan \\
\hline 5 & EC-802558 & AVRDC, Taiwan \\
\hline 6 & EC-802559 & AVRDC, Taiwan \\
\hline 7 & EC-802560 & AVRDC, Taiwan \\
\hline 8 & EC-802561 & AVRDC, Taiwan \\
\hline 9 & EC-802562 & AVRDC, Taiwan \\
\hline 10 & EC-802563 & AVRDC, Taiwan \\
\hline 11 & Pusa Ruby & IARI, New Delhi \\
\hline 12 & Pusa Uphar & IARI, New Delhi \\
\hline 13 & Pusa Sadabahr & IARI, New Delhi \\
\hline 14 & Pusa-120 & IARI, New Delhi \\
\hline 15 & Pusa Sheetal & IARI, New Delhi \\
\hline 16 & Pusa Rohini & IARI, New Delhi \\
\hline 17 & Arka Alok & IIHR, Banglore \\
\hline 18 & Arka Abha & IIHR, Banglore \\
\hline 19 & Arka Vikas & IIHR, Banglore \\
\hline 20 & Punjab Chhuhara & PAU, Ludhiana \\
\hline 21 & Pant T-3 & GBPUT, Pantnagar \\
\hline 22 & LC-1 & UHF, Nauni \\
\hline 23 & LC-2 & Solan \\
\hline 24 & LC-3 & \\
\hline 25 & Solan Lalima (Check) & \\
\hline & & \\
\hline
\end{tabular}


Table.2 Mean performance of tomato genotypes for different horticultural traits.

\begin{tabular}{|c|c|c|c|c|c|c|c|c|}
\hline Genotypes & $\begin{array}{l}\text { Days to } \\
\text { first fruit } \\
\text { harvesting }\end{array}$ & $\begin{array}{c}\text { Plant } \\
\text { height } \\
\text { (cm) }\end{array}$ & $\begin{array}{c}\text { Fruit } \\
\text { length } \\
\text { (cm) }\end{array}$ & $\begin{array}{c}\text { Fruit } \\
\text { breadt } \\
\text { h }(\mathrm{cm})\end{array}$ & $\begin{array}{l}\text { Average } \\
\text { fruit } \\
\text { weight } \\
\text { (g) }\end{array}$ & $\begin{array}{l}\text { Number } \\
\text { of fruit } \\
\text { clusters } \\
\text { per plant }\end{array}$ & $\begin{array}{c}\text { Number } \\
\text { of fruits } \\
\text { per } \\
\text { cluster }\end{array}$ & $\begin{array}{c}\text { Number } \\
\text { of fruits } \\
\text { per } \\
\text { plant }\end{array}$ \\
\hline EC-802554 & 66.00 & 63.25 & 5.17 & 5.33 & 63.39 & 5.37 & 4.03 & 21.64 \\
\hline EC-802555 & 65.33 & 76.87 & 3.53 & 3.40 & 30.08 & 6.07 & 5.05 & 30.60 \\
\hline EC-802556 & 66.33 & 64.63 & 5.16 & 5.25 & 62.34 & 6.40 & 3.72 & 23.80 \\
\hline EC-802557 & 75.00 & 69.90 & 5.57 & 5.70 & 70.17 & 6.43 & 3.73 & 24.00 \\
\hline EC-802558 & 71.00 & 68.67 & 5.24 & 5.29 & 63.77 & 5.93 & 2.96 & 17.57 \\
\hline EC-802559 & 73.00 & 66.08 & 5.20 & 4.70 & 56.18 & 6.60 & 2.58 & 17.02 \\
\hline EC-802560 & 74.00 & 69.28 & 5.09 & 4.67 & 54.69 & 5.67 & 3.18 & 18.01 \\
\hline EC-802561 & 71.67 & 74.07 & 5.63 & 5.44 & 70.44 & 5.40 & 2.93 & 15.84 \\
\hline EC-802562 & 63.33 & 67.60 & 5.89 & 5.65 & 76.65 & 6.87 & 2.92 & 20.03 \\
\hline EC-802563 & 72.33 & 77.83 & 5.87 & 5.55 & 74.91 & 7.20 & 2.31 & 16.62 \\
\hline Pusa Ruby & 64.00 & 98.87 & 3.58 & 4.97 & 40.90 & 5.57 & 4.48 & 24.90 \\
\hline Pusa Uphar & 68.00 & 65.93 & 4.90 & 5.33 & 60.07 & 3.60 & 5.65 & 20.27 \\
\hline Pusa Sadabhar & 64.33 & 63.14 & 4.84 & 5.01 & 55.82 & 5.40 & 3.80 & 20.53 \\
\hline Pusa-120 & 76.33 & 72.27 & 4.06 & 5.10 & 47.70 & 5.47 & 3.64 & 19.83 \\
\hline Pusa Sheetal & 64.67 & 61.13 & 4.42 & 5.12 & 52.08 & 5.43 & 3.63 & 19.70 \\
\hline Pusa Rohini & 81.33 & 65.90 & 5.23 & 4.80 & 57.74 & 5.70 & 4.29 & 24.43 \\
\hline Arka Alok & 68.67 & 55.73 & 5.52 & 5.96 & 75.62 & 5.17 & 4.06 & 21.00 \\
\hline Arka Abha & 74.67 & 75.27 & 5.20 & 5.26 & 62.97 & 5.60 & 4.07 & 22.60 \\
\hline Arka Vikas & 74.33 & 70.40 & 5.63 & 5.32 & 68.85 & 5.37 & 3.78 & 20.27 \\
\hline Punjab Chhuhara & 62.33 & 67.63 & 5.61 & 4.23 & 59.40 & 5.53 & 4.27 & 23.60 \\
\hline Pant T-3 & 73.67 & 73.00 & 3.79 & 4.66 & 40.61 & 5.70 & 3.84 & 21.90 \\
\hline LC-1 & 73.67 & 71.63 & 4.33 & 4.39 & 43.76 & 4.47 & 3.48 & 15.53 \\
\hline LC-2 & 62.00 & 87.96 & 5.09 & 5.75 & 67.35 & 6.50 & 3.67 & 23.87 \\
\hline LC-3 & 69.00 & 75.31 & 4.18 & 2.64 & 25.37 & 4.73 & 3.60 & 17.07 \\
\hline $\begin{array}{l}\text { Solan Lalima } \\
\text { (Check) }\end{array}$ & 67.33 & 86.95 & 5.43 & 5.23 & 65.32 & 5.10 & 4.43 & 22.58 \\
\hline Mean & 69.69 & 71.57 & 4.97 & 4.99 & 57.85 & 5.65 & 3.76 & 20.93 \\
\hline Range & $\begin{array}{l}62.00- \\
81.33\end{array}$ & $\begin{array}{c}\mathbf{5 5 . 7 3 -}- \\
\mathbf{9 8 . 8 7}\end{array}$ & $\begin{array}{c}3.53- \\
5.89\end{array}$ & $\begin{array}{c}2.64- \\
5.96\end{array}$ & $\begin{array}{c}25.37- \\
76.65\end{array}$ & $\begin{array}{c}3.60- \\
7.20\end{array}$ & $\begin{array}{c}2.31- \\
5.65\end{array}$ & $\begin{array}{c}15.53- \\
30.60\end{array}$ \\
\hline$\pm \mathrm{SE}(\mathrm{m})$ & 0.932 & 1.385 & 0.123 & 0.107 & 1.946 & 0.153 & 0.162 & 0.740 \\
\hline $\mathrm{CV}(\%)$ & 2.317 & 3.351 & 4.293 & 3.721 & 5.826 & 4.691 & 7.466 & 6.126 \\
\hline C.D. (0.05) & 2.659 & 3.950 & 0.351 & 0.306 & 5.550 & 0.437 & 0.463 & 2.111 \\
\hline
\end{tabular}


Table.3 Mean performance of tomato genotypes for various horticultural traits.

\begin{tabular}{|c|c|c|c|c|c|c|c|c|}
\hline Genotypes & $\begin{array}{l}\text { Number } \\
\text { of locules } \\
\text { per Fruit }\end{array}$ & $\begin{array}{c}\text { Harvest } \\
\text { duration } \\
\text { (days) }\end{array}$ & $\begin{array}{l}\text { Shelf life } \\
\text { (days) }\end{array}$ & $\begin{array}{c}\text { Pericarp } \\
\text { thickness } \\
(\mathbf{m m})\end{array}$ & $\begin{array}{c}\text { Total } \\
\text { soluble } \\
\text { solids } \\
\left({ }^{\circ} \text { Brix }\right)\end{array}$ & $\begin{array}{c}\text { Buckeye rot } \\
\text { incidence }(\%)^{*}\end{array}$ & $\begin{array}{l}\text { Marketa } \\
\text { ble fruit } \\
\text { yield per } \\
\text { plant (kg) }\end{array}$ & $\begin{array}{l}\text { Marketab } \\
\text { le fruit } \\
\text { yield per } \\
\text { hectare } \\
\text { (q) }\end{array}$ \\
\hline EC-802554 & 3.00 & 38.00 & 24.33 & 7.37 & 4.77 & $23.61(23.61)$ & 1.37 & 406.42 \\
\hline EC-802555 & 2.33 & 46.67 & 28.00 & 7.18 & 4.87 & $26.42(26.43)$ & 0.92 & 272.40 \\
\hline EC-802556 & 3.67 & 41.33 & 25.00 & 6.41 & 4.67 & $24.10(24.11)$ & 1.48 & 438.68 \\
\hline EC-802557 & 4.33 & 34.00 & 30.33 & 7.78 & 5.03 & $23.81(23.82)$ & 1.68 & 498.90 \\
\hline EC-802558 & 4.33 & 26.00 & 22.00 & 6.67 & 4.43 & $28.30(28.31)$ & 1.12 & 332.19 \\
\hline EC-802559 & 3.67 & 27.00 & 19.33 & 5.86 & 4.70 & $29.62(29.63)$ & 0.96 & 283.68 \\
\hline EC-802560 & 2.67 & 29.67 & 20.67 & 6.26 & 4.80 & $26.45(26.46)$ & 0.98 & 291.26 \\
\hline EC-802561 & 3.33 & 23.00 & 22.00 & 6.67 & 4.37 & $28.13(28.14)$ & 1.12 & 330.21 \\
\hline EC-802562 & 3.00 & 35.00 & 31.33 & 7.85 & 4.43 & $26.91(26.92)$ & 1.54 & 454.84 \\
\hline EC-802563 & 3.67 & 21.33 & 22.67 & 6.87 & 4.80 & $28.51(28.53)$ & 1.25 & 369.07 \\
\hline Pusa Ruby & 3.00 & 39.33 & 21.67 & 6.57 & 3.65 & $27.27(27.28)$ & 1.02 & 301.76 \\
\hline Pusa Uphar & 3.00 & 30.00 & 24.00 & 7.27 & 3.77 & $27.22(27.23)$ & 1.21 & 360.18 \\
\hline $\begin{array}{c}\text { Pusa } \\
\text { Sadabhar }\end{array}$ & 3.33 & 23.33 & 22.67 & 6.87 & 4.10 & $27.50(27.51)$ & 1.15 & 339.93 \\
\hline Pusa-120 & 3.00 & 26.00 & 20.33 & 6.16 & 4.53 & $24.44(24.45)$ & 0.95 & 279.87 \\
\hline Pusa Sheetal & 3.00 & 29.33 & 21.67 & 6.56 & 4.37 & $24.09(24.10)$ & 1.02 & 303.32 \\
\hline Pusa Rohini & 3.00 & 42.00 & 21.33 & 6.46 & 4.83 & $23.88(23.89)$ & 1.41 & 417.98 \\
\hline Arka Alok & 3.67 & 21.33 & 20.33 & 6.16 & 4.47 & $27.06(27.07)$ & 1.59 & 470.77 \\
\hline Arka Abha & 5.67 & 25.00 & 19.00 & 5.76 & 5.00 & $24.05(24.06)$ & 1.42 & 421.79 \\
\hline Arka Vikas & 5.00 & 31.67 & 16.33 & 4.95 & 3.93 & $22.32(22.33)$ & 1.39 & 412.87 \\
\hline $\begin{array}{c}\text { Punjab } \\
\text { Chhuhara }\end{array}$ & 4.67 & 32.33 & 23.00 & 7.42 & 4.77 & $24.20(24.21)$ & 1.40 & 415.09 \\
\hline Pant T-3 & 3.33 & 27.67 & 16.00 & 4.85 & 4.80 & $30.03(30.04)$ & 0.89 & 263.38 \\
\hline LC-1 & 3.33 & 27.67 & 16.33 & 4.95 & 3.90 & $33.41(33.42)$ & 0.68 & 201.02 \\
\hline LC-2 & 4.33 & 36.33 & 29.00 & 7.63 & 5.10 & $20.05(20.06)$ & 1.61 & 476.27 \\
\hline LC-3 & 3.33 & 24.00 & 14.00 & 4.24 & 4.07 & $33.04(33.05)$ & 0.44 & 128.83 \\
\hline $\begin{array}{c}\text { Solan } \\
\text { Lalima } \\
\text { (Check) }\end{array}$ & 4.33 & 39.00 & 22.00 & 6.67 & 4.73 & $24.18(24.19)$ & 1.47 & 436.42 \\
\hline Mean & 3.60 & 31.08 & 22.13 & 6.46 & 4.52 & $26.34(26.35)$ & 1.20 & 356.29 \\
\hline Range & $\begin{array}{c}2.33- \\
5.67\end{array}$ & $\begin{array}{c}21.33- \\
46.67\end{array}$ & $\begin{array}{c}14.00- \\
31.33\end{array}$ & $\begin{array}{c}4.24- \\
7.85\end{array}$ & $3.65-5.10$ & $20.06-33.41$ & $\begin{array}{c}0.44- \\
1.68\end{array}$ & $\begin{array}{c}128.83- \\
498.90\end{array}$ \\
\hline$\pm \mathrm{SE}(\mathrm{m})$ & 0.303 & 0.888 & 0.711 & 0.210 & 0.144 & 0.796 & 0.050 & 14.91 \\
\hline $\mathrm{CV}(\%)$ & 14.596 & 4.950 & 5.565 & 5.626 & 5.517 & 5.230 & 7.237 & 7.25 \\
\hline C.D. (0.05) & 0.865 & 2.534 & 2.028 & 0.598 & 0.410 & 2.269 & 0.143 & 42.52 \\
\hline
\end{tabular}

* Figures in the parenthesis are Arc-Sine transformed values 
Table.4 Estimates of phenotypic and genotypic coefficients of variation, heritability, genetic advance and genetic gain for various characters in tomato

\begin{tabular}{|c|c|c|c|c|c|c|c|}
\hline \multirow[t]{2}{*}{ Characters } & \multirow[t]{2}{*}{ Range } & \multirow{2}{*}{$\begin{array}{l}\text { Mean } \pm \\
\text { SE }(d)\end{array}$} & \multicolumn{2}{|c|}{$\begin{array}{l}\text { Coefficients of } \\
\text { Variability }(\%)\end{array}$} & \multirow[t]{2}{*}{$\begin{array}{c}\text { Heritabilit } \\
\text { y }(\%)\end{array}$} & \multirow[t]{2}{*}{$\begin{array}{l}\text { Genetic } \\
\text { advance }\end{array}$} & \multirow[t]{2}{*}{$\begin{array}{c}\text { Genetic } \\
\text { gain }(\%)\end{array}$} \\
\hline & & & Genotypic & Phenotypic & & & \\
\hline $\begin{array}{l}\text { Days to first fruit harvesting } \\
\text { (number) }\end{array}$ & $62.00-81.33$ & $\begin{array}{l}69.69 \pm \\
1.32\end{array}$ & 7.18 & 7.54 & 90.57 & 9.81 & 14.08 \\
\hline Plant height $(\mathrm{cm})$ & $55.73-98.87$ & $\begin{array}{l}71.57 \pm \\
1.96\end{array}$ & 12.75 & 13.19 & 93.54 & 18.18 & 25.41 \\
\hline Fruit length $(\mathrm{cm})$ & $3.53-5.89$ & $4.97 \pm 0.17$ & 13.88 & 14.53 & 91.27 & 1.36 & 27.32 \\
\hline Fruit breadth $(\mathrm{cm})$ & $2.64-5.96$ & $4.99 \pm 0.15$ & 14.55 & 15.02 & 93.87 & 1.45 & 29.04 \\
\hline Average fruit weight (g) & $25.37-76.65$ & $\begin{array}{l}57.85 \pm \\
2.75\end{array}$ & 23.22 & 23.94 & 94.08 & 26.83 & 46.39 \\
\hline Number of fruit clusters per plant & $3.60-7.20$ & $5.65 \pm 0.22$ & 13.40 & 14.19 & 89.07 & 1.47 & 26.04 \\
\hline Number of fruits per cluster & $2.31-5.65$ & $3.76 \pm 0.23$ & 19.07 & 20.48 & 86.71 & 1.38 & 36.59 \\
\hline Number of fruits per plant & $15.53-30.60$ & $\begin{array}{l}20.93 \pm \\
1.05\end{array}$ & 16.23 & 17.35 & 87.53 & 6.55 & 31.29 \\
\hline Number of locules per fruit & $2.33-5.67$ & $3.60 \pm 0.43$ & 20.19 & 24.91 & 65.68 & 1.21 & 33.71 \\
\hline Harvest duration (days) & $21.33-46.67$ & $\begin{array}{l}31.08 \pm \\
1.26\end{array}$ & 22.57 & 23.10 & 95.41 & 14.11 & 45.41 \\
\hline Shelf life (days) & $14.00-31.33$ & $\begin{array}{c}22.13 \pm \\
1.01\end{array}$ & 19.29 & 20.08 & 92.32 & 8.45 & 38.19 \\
\hline Pericarp thickness (mm) & $4.24-7.85$ & $6.46 \pm 0.30$ & 14.33 & 15.40 & 86.65 & 1.78 & 27.48 \\
\hline Total soluble solids ( ${ }^{\circ}$ Brix) & $3.65-5.10$ & $4.52 \pm 0.20$ & 8.47 & 10.11 & 70.20 & 0.66 & 14.61 \\
\hline Buckeye rot incidence (\%) & $20.06-33.41$ & $\begin{array}{l}26.34 \pm \\
1.12\end{array}$ & 11.58 & 12.71 & 83.06 & 5.73 & 21.75 \\
\hline Marketable fruit yield per plant $(\mathrm{kg})$ & $0.44-1.68$ & $1.20 \pm 0.07$ & 25.41 & 26.42 & 92.50 & 0.61 & 50.35 \\
\hline
\end{tabular}


Ten genotypes recorded maximum value for total soluble solids than the standard check cultivar Solan Lalima $\left(4.73{ }^{\circ} \mathrm{B}\right)$. Results of the present study were in line with the findings of Meena et al., (2018), Singh et al., (2017a), Rai et al., (2016), Patel et al., (2013), Meena et al., (2015) and Reddy et al., (2013a).

\section{Buckeye rot incidence (\%)}

In the mid hills zones of Himachal Pradesh, buckeye rot of tomato is one of the most destructive disease. The incidence ranged from 20.05 to $33.41 \%$ (Table 3 ) and varied significantly among all the genotypes under study. Buckeye rot incidence was minimum in LC-2 (20.05\%) which was statistically at par with Arka Vikas (22.32\%). The values were rated as moderately susceptible as per the scale given by Dodan et al., (1995). The buckeye rot incidence was comparatively less (20.05-24.10 \%) in eight genotypes than the standard check cultivar Solan Lalima (24.18 $\%$ ), whereas, maximum incidence of disease was observed in LC-1 (33.41\%). Similar findings were also reported by Kumar et al., (2006).

Marketable fruit yield per plant (kg) and per hectare (q)

Fruit yield $(\mathrm{kg} /$ plant and $\mathrm{q} / \mathrm{ha}$ ranged from $0.44-1.68 \mathrm{~kg} /$ plant and128.83- 498.90q/ha (Table 3) and varied significantly among all the genotypes under study (Appendix-I). General average for this attribute was $1.20 \mathrm{~kg} / \mathrm{plant}$ and $356.29 \mathrm{q} / \mathrm{ha}$. EC-802557 $(1.68 \mathrm{~kg} / \mathrm{plant}$ and $498.90 \mathrm{q} / \mathrm{ha})$ reported the maximum fruit yield and minimum fruit yield was recorded in LC-3 $(0.44 \mathrm{~kg} /$ plant and $128.83 \mathrm{q} / \mathrm{ha}$ ). Five genotypes recorded higher yield (kg/plant and $\mathrm{q} / \mathrm{ha}$ ) than the standard check cultivar Solan Lalima $(1.47 \mathrm{~kg} /$ plant and $436.42 \mathrm{q} / \mathrm{ha}$ ). Similar observations were made by Meena et al., (2018), Singh et al., (2017a) and Patel et al., (2013).

\section{Parameters of Variability}

To assess the prevailing variation in the studied genotypes of tomato, the estimates of variability were worked out for various characters after analyzing the data recorded for different horticultural characters and which are presented in Table 4. It was observed that the major variation was because of the effect of genotype and environment.

\section{Coefficients of variability}

For all the characters studied, phenotypic coefficients of variation were higher in magnitude than the corresponding genotypic coefficients of variation, though the difference was less in majority of cases thus, indicating that environmental factors have played less influence on the expression of these characters.

\section{Phenotypic and genotypic coefficients of variation}

The perusal of data (Table 4) revealed marked extent of variation for all the characters studied. It ranged from $7.54 \%-26.42 \%$ at phenotypic level and $7.18 \%-25.41 \%$ at genotypic level.

The phenotypic and genotypic coefficients of variability were high for average fruit weight (23.94\% and $23.22 \%$ ), number of locules per fruit $(24.91 \%$ and $20.19 \%$ ), harvest duration (23.10\% and $22.57 \%)$ and marketable fruit yield per plant $(26.42 \%$ and $25.41 \%)$. Similar findings have been reported by Rai et al., (2016), Bhandari et al., (2017) and Meena et al., (2018).

Moderate coefficients of variability both at phenotypic and genotypic level were observed for plant height $(13.19 \%$ and $12.75 \%)$, fruit length (14.53\% and $13.88 \%)$, fruit breadth 
(15.02\% and $14.55 \%)$, number of fruit clusters per plant $(14.19 \%$ and $13.40 \%)$, number of fruits per plant $(17.35 \%$ and $16.23 \%)$, pericarp thickness $(15.40 \%$ and $14.33 \%)$ and buckeye rot incidence (12.71\% and $11.58 \%)$.

Similar findings were reported by Bhandari et al., (2017) and Meena et al., (2018). Low coefficients of variability was observed for days to first fruit harvesting $(7.54 \%$ and $7.18 \%$ ) as also confirmed from the findings of Reddy et al., (2018).

High coefficients of variability at phenotypic level along with moderate coefficients of variability at genotypic level were recorded for number of fruits per cluster $(20.48 \%$ and $19.07 \%)$ and shelf life (20.08\% and $19.29 \%)$.

Moderate coefficient of variability at phenotypic level along with low coefficient of variability at genotypic level was recorded for total soluble solids $(10.11 \%$ and $8.47 \%)$ as also confirmed from the findings of Meena $e t$ al., (2018)

\section{Heritability}

Heritability in broad sense is a measure of the proportions of phenotypic variance resulting due to genetic factors. Heritability (broad sense) estimates ranged from $65.68-95.41 \%$ (Table 4).

High heritability was recorded for harvest duration $(95.41 \%)$, average fruit weight (94.08\%), fruit breadth (93.87\%), plant height (93.54\%), marketable fruit yield per plant $(92.50 \%)$, shelf life $(92.32 \%)$, fruit length (91.27\%), days to first fruit harvesting (90.57\%), pericarp thickness $(86.65 \%)$, buckeye rot incidence (83.06\%), total soluble solids $(70.20 \%)$ and number of locules per fruit $(65.68 \%)$ which were in accordance with the findings of Rai et al., (2016) for marketable fruit yield per plant, harvest duration, days to first fruit harvesting, total soluble solids, number of locules per fruit, pericarp thickness, plant height, average fruit weight. Similarly Reddy et al., (2018) reported for number of fruits per plant, total soluble solids, number of fruit clusters per plant and marketable fruit yield per plant.

\section{Genetic advance and genetic gain}

The genetic gain ranged from 14.08 to $50.35 \%$ (Table 4) and was moderate to high in nature. High genetic gain was recorded for marketable fruit yield per plant $(50.35 \%)$, average fruit weight $(46.39 \%)$, harvest duration $(49.41 \%)$, shelf life $(38.19 \%)$, number of fruits/cluster (36.59\%), number of locules/fruit (33.71\%), number of fruits/plant (31.29\%), fruit breadth (29.04\%), pericarp thickness (27.48\%), fruit length $(27.32 \%)$, number of fruit clusters/plant (26.04\%), plant height $(25.41 \%)$ and buckeye rot incidence $(21.75 \%)$, while it was moderate for total soluble solids $(14.61 \%)$ and days to first fruit harvesting (14.08\%) which were in accordance with the findings of Reddy et al., (2018), Shankar et al., (2013) and Prajapati et al., (2015).

On the basis of overall performance, out of 25 genotypes, EC-802557, LC-2 and Arka Alok were found superior for marketable fruit yield and other important characters. The estimates of PCV and GCV were high for marketable fruit yield/plant, number of locules/fruit, average fruit weight, harvest duration, shelf life and number of fruits/cluster.

High heritability was observed for all the characters among all genotypes. Genetic advance was observed high for marketable fruit yield per plant, average fruit weight, harvest duration, shelf life, number of fruits/cluster, number of locules/fruit, number of fruits/plant, fruit breadth, pericarp thickness, number of fruit cluster/plant, fruit length and plant height. 


\section{References}

Allard, R.W. 1960. Principles of Plant Breeding. John Wiley and sons, New York. 485p

Anonymous, 2016-17(b). Indian Horticulture Database. National Horticulture Board, Ministry of Agriculture, Government of India, Gurgaon, pp. 219.

Anonymous, 2018-19(a). Indian Horticulture Database. National Horticulture Board, Ministry of Agriculture, Government of India.

Bhandari, H.R., Srivastava, K. and Reddy, G.E. 2017. Genetic variability, heritability and genetic advance for yield traits in tomato (Solanum lycopersicum L.). International Journal of Current Microbiology and Applied Sciences 6(7): 4131-4138.

Bose, T. K., Bose, J., Kabir, T. K., Maity, V. A., Parthasarathy and Som, M. G. 2002. Vegetable crops. Bhumari Mitra Publication, Kolkata, India.

Burton, G.W. and De Vane, E. W. 1953. Estimating heritability in tall fescue (Festuca arundiancea) from replicated clonal material. Proejtunniens 9(22): 1215.

Clinton, S. K. 2005. Tomatoes or lycopene: a role in prostate carcinogenesis. Journal of Nutrition 135(8): 2057-2059.

Dodan, D. S., Shyam, K. P. and Bhardwaj, S. S. 1995. Relative responses of tomato cultivar lines against Buckeye rot under field conditions. Pl. Dis. Res. 10(2): 135136.

Gomez, K. A. and Gomez, A. A. 1983. Statistical Procedures for Agricultural Research. John Wiley and Sons Inc., New York. pp. 357-427.

Johnson, H.W., Robinson, H. F. and Comstock, R. E. 1955. Estimates of genetic and environmental variability in soybean. Agronomy Journal 47: 314-318.

Meena, R.K., Kumar, S., Meena, M. L. and Verma, S. 2018. Genetic variability, heritability and genetic advance for yield and quality attributes in tomato. Journal of Pharmacognosy and Phytochemistry 7(1):1937-1939.

Patel, S.A., Kshirsagar, D.B., Attar, A.V. and Bhalekar, M.N. 2013. Study on genetic variability, heritability and genetic advance in tomato. International Journal of Plant Sciences 8(1): 45-47.

Prajapati, S., Tiwari, A., Kadwey, S. and Jamkar, T. 2015. Genetic variability, heritability and genetic advance in tomato. International Journal of Agriculture and Biotechnology 8(2): 245-251.

Rai, A.K., Vikram, A. and Pandav, A. 2016. Genetic variability studies in tomato for yield and quality traits. International Journal of Agriculture, Environment and Biotechnology 9(5): 739-744.

Reddy, B.R., Reddy, S.D., Reddaiah, K. and Sunil, N. 2013(a). Studies on genetic variability, heritability and genetic advance for yield and quality traits in tomato. International Journal of Current Microbiology and Applied Sciences 2(9): 238-244.

Shankar, A., Reddy, R.V. S. K., Sujatha, M. and Pratap, M. 2013. Genetic variability studies in $\mathrm{F}_{1}$ generation of tomato. Journal of Agriculture and Veterinary Science 4(5): 31-34.

Singh, A.K., Ram, C.N., Yadav, G.C., Srivastava, R.K., Deo, C., Gautam, D.K., Kumar, P. and Kumar, P. 2017(a). Studies on genetic variability, heritability and genetic advance in tomato. International Journal of Pure and Applied Bioscience 5(2): 908912.

\section{How to cite this article:}

Dharminder Kumar, Achal Kashyap, Balbir Singh Dogra, Kumud Jaryal, Atul Gupta, Sandeep Kumar, Aanchal Chauhan, Kumari Shiwani and Rajesh Kaler. 2021. Genetic evaluation of tomato (Solanum lycopersicum L.) germplasm for yield and quality traits. Int.J.Curr.Microbiol.App.Sci. 9(11): 3848-3859. doi: https://doi.org/10.20546/ijcmas.2020.911.460 